\title{
The perfect PARTNER: no HIV transmission with ART
}

\section{$c$}

an HIV-positive person with an undetectable viral load does not transmit HIV to their partner

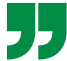

The results of the second phase of the PARTNER study PARTNER2 - have been published in The Lancet. PARTNER2 assessed the risk of HIV transmission via condomless sex in serodifferent men who have sex with men (MSM) in a partnership when the HIV-positive partner was taking antiretroviral therapy (ART). The transmission rate recorded was zero when HIV viral load was effectively suppressed, supporting the message that undetectable equals untransmittable $(\mathrm{U}=\mathrm{U})$, which is that an HIV-positive person with an undetectable viral load does not transmit HIV to their partner.

In the first phase of the prospective, observational PARTNER study, 1,166 HIV-serodifferent couples were enrolled; overall, 888 couples

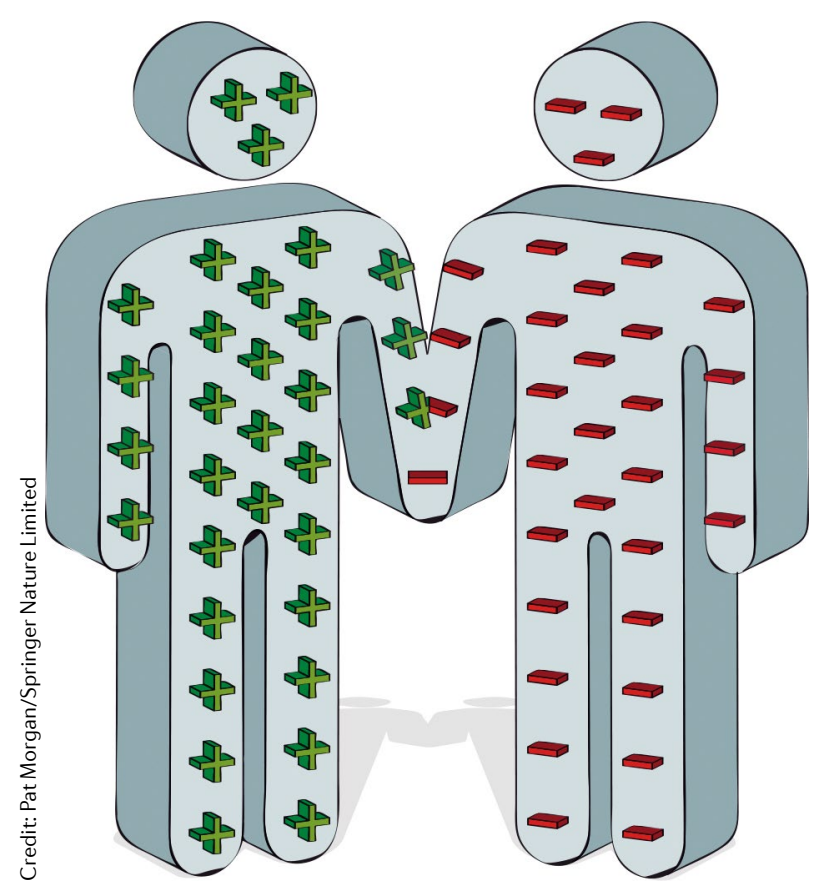

(548 heterosexual couples and 340 gay couples) provided 1,238 eligible couple-years for analysis. Couples reported condomless sex for a median of 2 years at baseline. During a median follow-up duration of 1.3 years, all couples reported condomless sex a median of 37 times per year.

In total, 11 of the HIV-negative partners became HIV-positive during eligible follow-up couple-years (1 heterosexual and 10 MSM); however, no phylogenetically linked transmissions occurred. Thus, the authors concluded that no incidents of within-couple HIV transmission occurred in phase 1 of the PARTNER study.

The second phase of the study, PARTNER2, recruited and monitored MSM only in order to increase the evidence level regarding the transmission risk of HIV in serodifferent MSM.

In PARTNER2, serodifferent MSM in a partnership were recruited from 75 clinical sites in 14 European countries. HIV-positive patients taking ART were asked if they had had recent condomless sex with an HIV-negative partner and if they were willing to take part in a transmission study. Overall, 972 couples were enrolled and 782 provided 1,593 eligible couple-years for follow-up analysis; median follow-up duration was 2 years.

HIV-positive partners had been taking ART for a median of 4.3 years with high adherence ( $98 \%$ of patients reporting $\geq 90 \%$ adherence) at baseline. In total, $97 \%$ of HIV-positive men had an undetectable viral load $(<50$ copies $/ \mathrm{ml})$ and $99 \%$ had a viral load of $<200$ copies $/ \mathrm{ml}$. Furthermore,
93\% of HIV-positive partners had a CD4 count $>350$ cells $/ \mu$ l. Only $5 \%$ of HIV-positive partners reported missing ART for $>4$ consecutive days during the follow-up period and adherence was $>90 \%$ in $92 \%$ of eligible couple-years. During the follow-up period, couples reported having condomless anal sex $\sim 76,088$ times; couples had condomless sex a median of 43 times/year.

During the study, 15 of the initially HIV-negative partners became HIV-positive. None of these incidences was phylogenetically linked to their partner's HIV. Of these men, 13 provided information on the potential source of their infection, 10 of whom reported recent condomless sex with men other than their partner in the study. Thus, the results of this study show that condomless anal sex with an HIV-positive partner taking ART with a viral load $<200$ copies $/ \mathrm{ml}$ results in an estimated transmission rate of zero.

These findings mean that the evidence regarding the risk of HIV transmission between serodifferent partners is now stronger for MSM than for heterosexuals. Together, the results of both phases of the PARTNER study reinforce the benefits of early testing for and treatment of HIV. Furthermore, these findings support the continued dissemination of the message of the $\mathrm{U}=\mathrm{U}$ campaign.

Louise Stone

ORIGINAL ARTICLE Rodger, A. J. et al. Risk of HIV transmission through condomless sex in serodifferent gay couples with the HIV-positive partner taking suppressive antiretroviral therapy (PARTNER): final results of a multicentre, prospective, observational study. Lancet. https:// doi.org/10.1016/S0140-6736(19)30418-0 (2019) 